\title{
THE NOVEMBER MEETING IN UNIVERSITY, ALABAMA
}

The five hundred and seventh meeting of the American Mathematical Society was held at the University of Alabama in University, Alabama on Friday and Saturday, November 26-27, 1954. About 130 persons registered, including 99 members of the Society.

By invitation of the Committee to Select Hour Speakers for Southeastern Sectional Meetings, Professor Nathan Jacobson of Yale University addressed the Society Friday evening on the subject Division rings, with Professor Alfred Brauer presiding.

Sessions for contributed papers were held Friday afternoon and Saturday morning, Professors A. S. Householder, G. B. Huff, Wallace Givens, A. D. Wallace, and F. A. Lewis presiding.

Abstracts of the papers presented follow. Those having the letter " $t$ " after their numbers were read by title. Where a paper has more than one author, that author whose name is followed by "(p)" presented it. Professor González was introduced by Professor F. A. Lewis, Dr. Swift by Professor J. H. Roberts, and Mr. Stallard by Professor W. M. Whyburn.

\section{Algebra ANd Theory of Numbers}

72. A. T. Brauer: Limits for the characteristic roots of a matrix. VII.

Some of the results of the former parts of this paper [I, Duke Math. J. vol. 13 (1946) pp. 387-395; II, ibid. vol. 14 (1947) pp. 21-26; III, ibid. vol. 15 (1948) pp. 871877; IV, ibid. vol. 19 (1952) pp. 75-91; V, ibid. vol. 19 (1952) pp. 553-562; VI, ibid. vol. 22 (1954) to be published] will be improved further by obtaining bounds for the components of the characteristic vectors. Let $A=\left(a_{k \lambda}\right)$ be an arbitrary matrix, $\omega$ a characteristic root, $\mathfrak{X}=\left(x_{1}, x_{2}, \cdots, x_{n}\right)$ a characteristic vector belonging to $\omega$, and $x_{r}$ the absolute greatest component. Then $\omega$ lies in the circle $C_{r}$ with center at $a_{r r}$ and radius $P_{r}=\sum_{\lambda \neq n}\left|a_{r \lambda}\right|$. Let $R$ be a closed subregion of $C_{r}$ containing $\omega$ and $d_{\kappa}$ be the minimum distance of $a_{k x}$ from $R$. Set $t_{r k}=1$ if $d_{k}=0$, and otherwise $t_{r k}=\min (1$, $\left.P_{\kappa} / d_{\kappa}\right)$ for $\kappa=1,2, \cdots, n ; \kappa \neq r$. It is shown that $\left|x_{\kappa}\right| \leqq t_{r \kappa}\left|x_{r}\right|$, and this result is improved by iteration. It follows that $\omega$ lies in the circle $\left|z-a_{r r}\right| \leqq \sum_{\lambda \neq n}\left|a_{r \lambda}\right| t_{r \lambda}$ contained in $C_{r}$. Moreover, if the inequalities for the $x_{k}$ contradict the $r$ th of the corresponding linear equations, then $\omega$ does not lie in $R$. If $A$ is non-negative, $\omega$ the greatest positive root, and $x_{m}$ the smallest component, then lower bounds for $x_{k} / x_{m}$ are obtained. Using these results it is of ten easy to compute $\omega$ as exactly as wanted. (Received October 13, 1954.)

\section{C. C. Buck: The algebraic aspect of integration in space.}

By "integration in space" is meant the $n$-dimensional analogue of the notions of integration along a curve, integration over a surface, etc. The term "algebraic" indicates that the discussion is restricted to topics that can be studied without the use of a limit process. An algebraic definition for space integrals has been improvised from a 
set of postulates for the notion of integral which were stated by Lebesgue in 1904 . (Received October 13, 1954.)

\section{4t. Leonard Carlitz: On a theorem of Stickelberger.}

Making use of a slight extension of the theorem $(D / p)=(-1)^{n-8}$, where $D$ is the discriminant of $f(x)$ and $f(x) \equiv g_{1}(x) \cdots g_{s}(x)(\bmod p)$, where the $g_{i}(x)$ are distinct irreducible polynomials, the following result is obtained. Let $D\left(a_{1}, \cdots, a_{n}\right)$ denote the discriminant of $f(x)=x^{n}+a_{1} x^{n-1}+\cdots+a_{n}$. Let $N$ denote the total number of solutions of $D\left(\xi_{1}, \cdots, \xi_{n}\right)=\eta^{2}, \xi_{i}, \eta \in G F(q), q$ odd, $N^{\prime}$ the number of solutions with $\eta \neq 0$. Then $N=q^{n-1}, N^{\prime}=q^{n}-q^{n-1}, n>1$. (Received October 4, 1954.)

\section{5t. Leonard Carlitz: Some arithmetic properties of elliptic functions.}

Making use of the familiar Fourier series for sn $x$, ns $x$, etc., it is shown in this paper that one can prove rapidly such results as Kummer's congruences for the coeffcients in the power series expansions (see Duke Math. J. vo.1 20 (1953) pp. 1-12). In the case of ns $x=\sum \beta_{m} x^{m} / m !$, an explicit relation connecting $\beta_{m}$ with the Bernoulli number $B_{m}$ leads to many properties of $\beta_{m}$, including some new ones. Moreover, congruences (of a restricted kind) are obtained for the coefficients of the Weierstrass A1-functions. (Received October 4, 1954.)

76t. Leonard Carlitz and J. H. Hodges: Representations by hermitian forms in a finite field.

Let $q=p^{n}, p>2$, and suppose that $\theta \in G F\left(q^{2}\right), \theta^{2}=v \in G F(q)$, but $\theta \notin G F(q)$. Then $\alpha=a+b \theta \in G F\left(q^{2}\right)$ if $a, b \in G F(q)$. By the conjugate of $\alpha$ is meant $\bar{\alpha}=a-b \theta$. If $A=\left(\alpha_{i j}\right)$ is a square matrix, $\alpha_{i j} \in G F\left(q^{2}\right)$, let $A^{*}=\bar{A}^{\prime}=\left(\bar{\alpha}_{i j}\right)^{\prime}$, where the prime denotes transpose. Then $A$ is hermitian if $A^{*}=A$. In this paper the authors determine the number $N_{t}(A, B)$ of $m \times t$ matrices $U$ such that $U^{*} A U=B$, where $A$ and $B$ are hermitian, $A$ is nonsingular of order $m, B$ is of order $t$ and rank $r \leqq m$, and all matrices have elements in $G F\left(q^{2}\right)$. Incidentally, the number $N(m, r)$ of hermitian matrices of order $m$ and rank $r$ is found. Also a certain exponential sum $H(B, s)$, which for $B=0$ reduces to $N(t, s)$, is considered. Using a relation between $N_{t}(A, B)$ and $H(B, s)$, they are able to explicitly evaluate the latter. They include an application of the sum $H(B, s)$ to the solution of a problem in partitions of hermitian matrices. It is worth noting that all of the results obtained hold as well if $A$ and $B$ are skew-hermitian $\left(A^{*}=-A\right.$, $B^{*}=-B$ ), for then the matrices $\theta A, \theta B$ are hermitian and may be used in place of $A$ and $B$ above. (Received October 4,1954 .)

\section{P. F. Conrad: Extensions of ordered groups.}

For definitions see Proc. Amer. Math. Soc. vol. 5 (1954) pp. 323-328. The factor group $C^{\prime} / C^{\prime \prime}$ of convex subgroups of an o-group $G$ where $C^{\prime}$ covers $C^{\prime \prime}$ is called a component of $G$. If the components of $G$ are $d$-closed, then there exists an $a$-closed $a$ extension of $G$ with each component $o$-isomorphic to the group $R$ of real numbers w.r.t. addition. A condition on the components of $G$ that is necessary and sufficient for $G$ to be $d$-closed is derived. Each $a$-closed $o$-group of rank 2 is $d$-closed and has both components $o$-isomorphic to $R$. Methods for construction of $o$-groups are given. (Received October 18, 1954.)

78. D. O. Ellis: Note on reflexive relations.

One studies the algebra of reflexive relations in a given set under the customary 
partial ordering and the generalized relational product recently employed in universal algebra (A. W. Goldie, The Jordan-Hölder theorem for general abstract algebras, Proc. London Math. Soc. vol. 52 (1950) pp. 107-131). One also shows that any such product of two reflexive relations gives rise to a partial ordering in a subset of the original set and that every such partial ordering may be obtained in this way. The methods are those of elementary set-theory and lattice-theory. (Received September 27, 1954.)

\section{Seymour Ginsburg (p) and J. R. Isbell: Certain real functions on Boolean algebras.}

Let $B$ be a complete Boolean algebra. $f$ is a quasi-simple function on $B$ if $f$ is a real-valued function, the domain of each value being a principal ideal of $B$. Multiplication and addition of two quasi-simple functions is defined. Let $S(B)\left(S^{*}(B)\right)$ be the set of all (bounded) quasi-simple functions, endowed with the uniform convergence topology $\left(S^{*}(B)\right.$ is normed in the usual manner). The ideal structure of the algebra $S(B)$ is intimately connected with the ideal structure of $B$. There is a one-one correspondence between the ideals of $S(B)$ and the ideals of $B$ which preserves inclusion, principal ideals, and prime ideals. Another sample result: an ideal in $S(B)$ is closed if and only if the corresponding ideal in $B$ is closed under countable unions. The ideal structure of $S^{*}(B)$ is less intimately bound to the ideal structure of $B$. The linear space conjugate to $S^{*}(B)$ is obtained. A necessary and then a sufficient condition for $S^{*}(B)$ to be complete, together with some examples, are given. (Received September 22, 1954.)

\section{0t. Seymour Ginsburg and J. R. Isbell: On a theorem of Sikorski.}

The pertinent results of R. Sikorski (Fund. Math. (1952)) are to the effect that if an $\aleph$-complete Boolean algebra $B$ is isomorphic to a $C$-additive field of sets, $F, C<\aleph$, then $B$ is isomorphic to some $\aleph$-additive field of sets. Here it is shown under somewhat weaker conditions that any such $F$ is already $\boldsymbol{N}$-additive. The point is contained in two observations: (1) a field representation of $B$ is $\boldsymbol{N}$-additive if and only if the two-valued measure on $B$ determined by each point in an element of the field is $\aleph$-additive; (2) the results of S. Ulam (Fund. Math. (1930)) on two-valued measures on complete atomic Boolean algebras apply for all Boolean algebras. A different proof of Ulam's results is given. (Received September 22, 1954.)

\section{E. H. Hadlock: On the equivalence of ternary quadratic forms.}

A necessary condition for a ternary quadratic form $f$ to be equivalent to a form $f^{\prime}$ with no term involving the product of distinct variables is that there exist a certain arithmetical invariant associated with $f$ and represented primitively by $f$. A necessary and sufficient condition for the equivalence of $f$ and $f^{\prime}$ is that there exist values of the variables for which the ternary quadratic form $U$ will be a common divisor of the three linear forms $Y_{i}$ for these same values of the variables. Moreover the coefficients of $U$ and of $Y_{i}$ are quadratic functions of $X_{i 3}, i=1,2,3$, and the coefficients of $X_{i 3}$ are the coefficients of $f$. Finally $X_{i 3}$ are three linear functions of $c_{j 3}, j=1,2,3$, associated with $f$, and $c_{j 3}$ are the elements of the third column of the transformation which takes $f$ into $f^{\prime}$. (Received October 13, 1954.)

\section{A. S. Householder: Norms of vectors and matrices.}

In the terminology of Faddeeva (Computational methods of linear algebra (in Russian), Moscow and Leningrad, 1950), a matrix norm $\|A\|$ is said to be consistent 
with a vector norm $\|x\|$ in case $\|A x\| \leqq\|A\| \cdot\|x\|$ for every $A$ and $x$, and to be subordinate in case for every $A$ there exists an $x \neq 0$ with the equality holding. Given an arbitrary vector $g$ of positive elements, one can define a vector norm $\|x\|_{\theta}$ (and another $\|x\|_{g^{\prime}}$ ) and a subordinate norm $\|A\|_{g}$ (or $\|A\|_{g^{\prime}}$ ). These have the property that, given a particular matrix $A$, if $|A|$ is the matrix of absolute values, and if $|A| g=g_{1}$, then $\|A\|_{\sigma^{\prime}} \leqq\|A\|_{\sigma}$. Such a property is desirable for sharpening convergence proofs and estimates of computational error. (Received August 27, 1954.)

\section{C. W. Huff: Existence of noncommuting matrices satisfying the logarithmic equation.}

John von Neumann has shown (Math. Zeit. vol. 30 (1929) p. 11) that when $A$ and $B$ commute and the norms of $A-I, B-I$, and $A B-I$ are all less than one, then $A$ and $B$ satisfy the $\operatorname{logarithmic}$ equation $\log A B=\log A+\log B$, where the norm of $A$ is $\left(A A^{*}\right)^{1 / 2}$. However, this paper shows that there are noncommuting matrices of any order greater than one satisfying the logarithmic equation by means of the following theorem, which is a consequence of previous results (Rend. Circ. Mat. Palermo (2) vol. 2 (1953) pp. 1-5): Let $f(w)=\left(e^{w}-1\right) / w$ for $w \neq 0$ and $f(0)=1$. If $A=\left(\begin{array}{c}0 \\ 0 \\ 0\end{array}\right)$ and $B=\left(\begin{array}{ll}0 & b \\ 0 & 0\end{array}\right)$ where $f(-z)=1$ and $b z \neq 0$, then the characteristic roots of $\exp A-I$, $\exp B-I$ and $\exp (A+B)-I$ are all less than one in absolute value. Furthermore, $\log (\exp A)(\exp B)=\log (\exp A)+\log (\exp B)$ and $(\exp A)(\exp B) \neq(\exp B)(\exp$ $A)$. (Received October 14, 1954.)

\section{W. V. Parker: A note on a theorem of Roth.}

In a recent paper, Roth (Proc. Amer. Math. Soc. vol. 5, pp. 1-3) proved the following theorem. If $A$ and $B$ are $n \times n$ matrices with elements in the field $F$, whose characteristic polynomials are $a_{0}\left(x^{2}\right)-x a_{1}\left(x^{2}\right)$ and $b_{0}\left(x^{2}\right)-x b_{1}\left(x^{2}\right)$ respectively, where $a_{0}(x), a_{1}(x), b_{0}(x)$, and $b_{1}(x)$ are elements in the polynomial domain, $F[x]$, of $F$; and if the rank of $A-B$ does not exceed unity, then the characteristic polynomial of $A B$ is $(-1)^{n}\left[a_{0}(x) b_{0}(x)-x a_{1}(x) b_{1}(x)\right]$. In this note the theorem is extended to give the characteristic polynomials of several other matrices which are polynomials in the matrices $A$ and $B$. (Received October 13, 1954.)

\section{P. B. Patterson: Almost regular forms}

Almost characteristics of the first, second, and third kinds are defined. Also defined are almost regular forms and almost regular forms of the first, second, and third classes. It is shown that there exist infinitely many genera containing at least two classes of positive forms possessing an almost characteristic of the second or third kind where the invariants associated with the forms may or may not contain a common odd prime factor to an odd power. The method used is similar to that employed by B. W. Jones and E. H. Hadlock (Proc. Amer. Math. Soc. vol. 4 (1953) pp. 539-543) to show primitive ternary indefinite quadratic genera of more than one class. (Received October 11, 1954.)

\section{B. M. Seelbinder: Bounds for positive solutions of pairs of linear Diophantine equations.}

Let $P$ be a $2 \times k$ matrix with positive integral elements $a_{i}(i=1,2, \cdots, k)$ in the first row and positive integral elements $b_{i}$ in the second row such that the elements of each row are relatively prime and such that the set of all minors $D_{i j}=a_{i} b_{j}-a_{j} b_{i}$ of order two has no common divisor. Assume that the columns are so arranged that 
$b_{1} / a_{1} \leqq b_{2} / a_{2} \leqq \cdots \leqq b_{k} / a_{k}$. Set $t_{i}=\left(a_{i}, b_{i}\right) ;$ let $L_{1}=D_{1 k}\left(D_{12}+D_{13}+\cdots+D_{1, k-1}\right) / t_{1} t_{k}$ and $L_{2}=D_{1 k}\left(D_{2 k}+D_{3 k}+\cdots+D_{k-1, k}\right) / t_{1} t_{k}$. It is proved that for every lattice point $(m, n)$ in the interior of that angular region which is bounded by the straight lines $a_{1} n-b_{1} m=L_{1}$ and $b_{k} m-a_{k} n=L_{2}$ and which lies completely in the first quadrant, the pair of linear Diophantine equations $a_{1} x_{1}+a_{2} x_{2}+\cdots+a_{k} x_{k}=m$ and $b_{1} x_{1}+b_{2} x_{2}$ $+\cdots+b_{k} x_{k}=n$ always has a solution in positive integers $x_{1}, x_{2}, \cdots, x_{k}$. (Received September 22, 1954.)

\section{7t. A. D. Wallace: The Green substructures of a compact connected mob.}

A mob is a Hausdorff space together with a continuous associative multiplication. Let $S$ be a compact connected mob. If $a \in S$ let $L_{a}=\{x \mid x \cup S x=a \cup S a\}$, see J. A. Green, Ann. of Math. (1950). Theorem. Let $K$ be the minimal ideal of $S$, let $a \in S \backslash K$, and let $C$ be the component of $S \backslash L_{a}$ containing $K$. Then $C^{*} \backslash C=L_{a}$. This result is related to a theorem due to Professor W. M. Faucett but the two theorems seem to be logically independent. If $R_{a}$ is defined dually and if $H_{a}=R_{a} \cap L_{a}$ then an analogous result holds for $H_{a}$. Hence we have the corollary: If $e$ is an idempotent not in $K$ and if $C$ is the component of $S \backslash H(e)$ containing $K$, then $H(e)=C^{*} \backslash C$. It should be observed that the conditions $\mathcal{X} C$ used by Green in his fundamental Theorem 8 do not hold for compact mobs. However, Green's proof mutatis mutandis is valid for a compact mob. Special cases of Green's Theorem 8 have been noted by Professor Faucett and Professor R. J. Koch in the compact case. (Received July 26, 1954.)

\section{8t. A. D. Wallace: The local structure of clans.}

A clan is a compact connected Hausdorff space together with a continuous associative multiplication with unit $u$. Let $S$ be one such and let $H(u)$ be the maximal subgroup of $S$ containing $u$. If $x \in S$ let $T(x)$ be the intersection of the sets $(S \backslash A)^{*}$ where $A$ runs through all subcontinua of $S$ not containing $x$. For each $x \in S$ the set $T(x)$ is a continuum (F. Burton Jones). Theorem: If $x \in H(u)$ and if $C$ is the component of $H(u)$ containing $x$ then $T(x) \subset C$. Corollary: If $H(u)$ is totally disconnected, then $S$ is semilocally connected at each $x \in H(u)$. It may happen that $H(u)$ is precisely the set of points where $S$ is semilocally connected. If $H(u)$ is connected then $S$ may be semilocally connected at no point. (Received July 26, 1954.)

\section{R. A. Willoughby: Almost singular systems of linear homo-} geneous equations. Preliminary report.

Given $A(\mu) \cdot x=0$ where $A(\mu)$ is an $n \times n$ ( $n=30$ app.) matrix whose elements are real-valued functions of a real variable $\mu$, the proposed problem is to find a computationally effective procedure for finding (approximately) (1) the least positive $\mu$ (if one such exists) for which $A(\mu)$ is singular and (2) the family of solution vectors $x$. It is assumed that one is given non-negative real numbers $\mu_{1}, \mu_{2}$ such that the desired $\mu$ is the only $\mu$ in the range $\mu_{1} \leqq \mu \leqq \mu_{2}$ for which $A(\mu)$ is singular. A plan of attack being pursued is to form $B(\mu)=A^{T}(\mu) \cdot A(\mu)$ and form an (approximately) equivalent triangular system using the method outlined in Goldstine and von Neumann's Numerical inverting of matrices of high order, Bull. Amer. Math. Soc. vol. 53 (1947) pp. 10211099. The desire is to choose $\mu$ so that at least one of the triangular diagonal elements is (almost) zero $(A(\mu)$ is so scaled that all the elements involved in the triangular system are less than one numerically). The resulting triangular system is then used to obtain the solution vectors. (Received October 4, 1954.) 


\section{ANALysis}

90. L. P. Burton: Conditions which preclude the existence of maximum, minimum, and minimax solutions of an ordinary differential system.

The system $y_{i+1}^{\prime}=f_{i+1}\left(x, y_{i}\right)(i=1, \cdots, n)$, where $y_{n+1}(x)$ and $f_{n+1}\left(x, y_{n}\right)$ are, by definition, $y_{1}(x)$ and $f_{1}\left(x, y_{n}\right)$ respectively, is considered. Let $R$ be a region in $(m+1)$ dimensional space and $R:\left(x_{0}, y_{10}, \cdots, y_{n 0}\right)$ be a point in $R$. By assuming continuity and certain monotone properties of the $f_{i}$ in the subsets of $R$ for which $x>x_{0}$ and $x<x_{0}$, it is shown that there cannot exist two distinct solutions $y_{1}(x), \cdots, y_{n}(x)$ and $z_{1}(x), \cdots, z_{n}(x)$, one of which has a limit point at $P$, the other of which approaches $P$ as a limit, which are such that for any $k, 1 \leqq k \leqq n, y_{k}(x) \neq z_{k}(x)$ over an interval $I: x_{0}<x<x_{0}+a, a^{2}>0$. More precisely, by assuming continuity of the $f_{i}$ in $R$ it follows that in the case of nonunique solutions through $P$ such solutions cannot be maximum, minimum, or minimax solutions. An extension is thus provided to the theory of critical solutions as originated by E. Kamke [Acta Math. vol. 58 (1932) pp. 57-85] and extended jointly by W. M. Whyburn and the author [Proc. Amer. Math. Soc. vol. 3 (1952) pp. 794-803]. It is further noted that the above general theorem gives a uniqueness theorem in case $n=1$ and, in case $n=2$, is closely related to the classical oscillation theory for the second-order differential system. (Received October 13, 1954.)

\section{1t. V. F. Cowling: Taylor methods of summation.}

In this paper the author obtains some new results regarding Taylor methods of summability and generalizes others previously obtained by Cowling and Piranian (Michigan Mathematical Journal vol. 1 (1952) pp. 73-78). Some typical results follow. Let $\left\{a_{n}\right\}$ be a sequence of complex numbers and suppose $\lim \sup \left|a_{n}\right| 1 / n=1 / R<\infty$ and let $\alpha$ be a real number $(0 \leqq \alpha<1 / 3, \alpha<R)$. If the series $\sum_{0}^{\infty} a_{n} n ! / z(z+1) \cdots(z+n)$ is absolutely summable $V_{\alpha}$ at $z_{0}$, it is absolutely summable $V_{\alpha}$ throughout the halfplane $\operatorname{Re}(z)>\operatorname{Re}\left(z_{0}\right)$. Similar results are obtained regarding ordinary summability of these and Newton series. The Euler and Taylor summability of series of the form $\sum_{0}^{\infty} a_{n} e^{-n_{z} \alpha_{z}}, 0<\alpha<1$, is also studied. (Received October 13, 1954.)

92. M. K. Fort, Jr.: Imbedding homeomorphisms in flows. Preliminary report.

Let $J$ be an interval of real numbers, and let $f$ be a continuously differentiable, order preserving homeomorphism of $J$ onto $J$. Conditions are found under which there exists a flow (one-parameter group of homeomorphisms) $F_{t},-\infty<t<\infty$, for which $F_{1}=f$ and for which each $F_{t}$ is continuously differentiable. It is shown that if $f$ has no fixed points except perhaps end points of $J$, and $f^{\prime}$ is positive-valued and nondecreasing, then: (i) if $J$ is an open interval, many such flows exist; (ii) if $J$ is a half open interval, a unique such flow exists; (iii) if $J$ is a closed interval, there may not exist such a flow. (Received October 11, 1954.)

\section{Tomlinson Fort: Solving linear difference equations by means of the Dirichlet series transform.}

The solution of linear differential equations by means of the Laplace transform is now a commonplace. This same transform can be used to solve linear difference equations with constant coefficients although the ideas are more complicated. A 
power series transformation has also been used for this same purpose. In the present paper it is shown how a Dirichlet series transform is a simpler and more natural instrument for solving difference equations. A table of transforms is made for the more common functions and illustrative equations are solved. (Received October 4, 1954.)

\section{Harry Gonshor: On uniformly closed rings generated by certain operators on a Hilbert space.}

Let $A$ be an operator which can be decomposed by direct integral theory onto twodimensional operators. Then with suitable identifications, it has been proved that for all such $A, A$ can be regarded as the identity function on a certain definite space $Z \cup_{Q}$ (the same for all such $A$ ), where $Z$ is the set of all complex numbers. To obtain the structure of the uniformly closed ring generated by $A$ and $A^{*}$, it is convenient to define the notion of the spectrum which generalizes the usual definition for normal operators. Then the ring is isomorphic and isometric to a subring of the ring of all functions which are continuous and second order matrix valued on $Q$, and continuous and complex-valued on $Z$. For a large class of such operators the subring may be taken to be the whole ring. (Received October 25, 1954.)

\section{M. O. González: Bilateral Laplace transformation of distribu-} tions.

If $q$ is a distribution, its bilateral Laplace transform $Q(s)$ ( $s$ complex) is defined and the fundamental rules of operational calculus are proved. Some transforms are given and also applications to the derivation of certain results involving the $\delta$ functional and to the solution of several extended integral equations of the form $f(t) * T=S$, where $f(t)$ is a given function and $S$ a given distribution. (Received October 13,1954.)

96. J. W. Jewett: Differentiable approximations to light interior transformations of plane domains. Preliminary report.

Let $D$ be a domain in the plane, let $n$ be a positive integer, and let a continuous mapping $f$ of $\bar{D}$ into the plane be light and strongly interior on $D$. Then there exists a sequence of continuous functions $f_{i}: \bar{D} \rightarrow E^{2}$ converging uniformly to $f$ on $\bar{D}$ such that each $f_{i}$ is light and strongly interior on $D$, agrees with $f$ on the boundary of $D$, and is $n$ times continuously differentiable on $D$. (Received October 18,1954.)

\section{W. B. Jurkat: The consistency of Nörlund and Hausdorff methods.}

Let $N, H$ denote the regular and real summability methods of Nörlund and Hausdorff respectively. Together with A. Peyerimhoff the following result is proved: If a series is $N$-summable to $s^{\prime}$ and $H$-summable to $s^{\prime \prime}$, then it is also summable in the generalized Abel sense to the common sum $s=s^{\prime}=s^{\prime \prime}$. In particular, the methods of Nörlund and Hausdorff are consistent as it was conjectured by E. Ullrich. A corresponding result holds for functional methods. (Research supported by U.S.A.F., Office of Scientific Research, Contract AF 18(600)-691.) (Received November 9, 1954.)

98. C. W. McArthur: Some applications of a theorem on uniform Cauchy points.

It is well known that the permutation group $P(N)$ of the natural numbers $N$ is of the second category relative to the topology induced on it by the Fréchet metric. Two 
theorems are proved which have as corollaries the following results. Suppose $(X,+, d)$ is a complete commutative semigroup with a pseudo metric $d$. Then a series $\sum_{i=1}^{\infty} x(i)$ in $X$ is unconditionally convergent if and only if $\left[t \in P(N):\left\{\sum_{i=1}^{n} x(t(i))\right\}\right.$ converges] is second category in $P(N)$. If $X$ is a weakly complete Banach space, $\sum_{i=1}^{\infty} x(i)$ is unconditionally convergent if and only if $\left[t \in P(N):\left\{\sum_{i=1}^{n} x(t(i))\right\}\right.$ is bounded] is second category. Suppose $s=\{s(i)\}$ is a sequence of real numbers. Let $E_{+\infty}(s)$ $=\left[t \in P(N): \limsup _{n} \sum_{i=1}^{n} s(t(i))=+\infty\right]$ and $E_{-\infty}(s)=\left[t \in P(N): \liminf _{n} \sum_{i=1}^{n} s(t(i))\right.$ $=-\infty]$. Then $E_{+\infty}(s) \cap E_{-\infty}(s)$ is a residual everywhere dense $G_{\delta}$ subset of $P(N)$ if and only if $E_{+\infty}(s)$ is non-null and $E_{-\infty}(s)$ is non-null. (Received August 13, 1954.)

\section{9t. J. S. MacNerney: Continuous products in linear spaces.}

Let $B$ denote the linear normed complete space of continuous linear transformations from a LNC space to itself. A quasi-harmonic operator is a function $M$ from the ordered number pairs $s, t$ to $B$ (for each $t$ ), of bounded variation in $s$ on each interval, such that (for each $s$ ) each of the limits $M(s, t-)$ and $M(s, t+)$ exists and such that $M(a, b) M(b, c)=M(a, c)$ and $M(a, a)=[M(a-, a)+M(a, a-)] / 2=[M(a, a+)$ $+M(a+, a)] / 2=1$ for each ordered number triple $a, b, c$. Extending the notion of a continuous product previously studied by the author (Stieltjes integrals in linear spaces, to appear in Ann. of Math.), a one-to-one correspondence, $M(s, t)={ }_{8} \prod t\{1$ $+d F\}$, is established between the class of quasi-harmonic operators $M$ and the class of functions $F$ from the real numbers to $B$, of bounded variation on each interval, such that $F(0)=0$ and $[F(s)-F(s-)]^{2}=[F(s+)-F(s)]^{2}=0$ for each number $s$. Application is made to continuous continued fractions and to certain discontinuous and nonlinear integral equations. (Received October 13, 1954.)

100. E. P. Miles, Jr. (p) and Ernest Williams: A basic set of homogeneous harmonic polynomials in $k$ variables.

For any set of non-negative integers $\left(b_{j}\right)$ such that $b_{1} \leqq 1$ and $\sum_{j=1}^{k} b_{j}=n$, let $M W_{b_{1}, b_{2}}^{n}, \cdots, b_{k}\left(x, x, \cdots, x_{k}\right)=\sum(-1)^{\left[a_{1} / 2\right]}\left(n !\left[a_{1} / 2\right] ! / \prod_{j=1}^{k} a_{j} ! \prod_{j=2}^{k}\left(\left(b_{j}-a_{j}\right) / 2\right) !\right)$ - $\prod_{j=1}^{k} x_{j} a_{j}$ where the summation extends over all $\left(a_{j}\right)$ such that $(1) a_{j} \equiv b_{j} \bmod 2$, $j=1,2, \cdots, k,(2) \sum_{j=1}^{k} a_{j}=n,(3) a_{j} \leqq b_{j}, j=2,3, \cdots, k$. The polynomials $M W$ form a basic set of homogeneous harmonic polynomials in $k$ variables. The authors modify them to form solutions of the generalized wave equation $\sum_{j=2}^{k} \partial^{2} u / \partial x_{j}^{2}$ $=\partial^{2} u / \partial x_{1}^{2}$ and the generalized Laplace equation $\sum_{j=1}^{k} \partial^{8} u / \partial x_{j}^{0}=0$. (Received October 4,1954 .)

101. E. P. Miles, Jr. and Ernest Williams (p): A basic set of homogeneous harmonic polynomials in $k$ variables. II.

It is shown that elements $M W_{b_{1}}^{n} \cdots b_{k}\left(x_{1} x_{2} \cdots x_{k}\right)$ of the author's basic set of homogeneous harmonic polynomials of degree $n$ in $k$ variables satisfy the following differential recursion formulas: (a) $\left(\partial / \partial x_{1}\right) M W_{1 b_{2}}^{n} \cdots b_{k}=n M W_{0 b_{2}}^{n-1} \cdots b_{k}, \quad 1+\sum_{j=2}^{k} b_{j}=n$; (b) $\left(\partial / \partial x_{j}\right) M W_{b_{1}} \cdots b_{j} \cdots b_{k}=n M W_{b_{1}}^{-1} \cdots b_{j}-1 \cdots b_{k}, j=2,3, \cdots, k, b_{1}=0,1, \sum_{j=1}^{m_{k}^{2}} b_{j}=n$; (c) $\left(\partial / \partial x_{1}\right) \quad M W_{0 b_{2}}^{n} \cdots b_{k}=-n \sum_{j=2}^{k} M W_{1 b_{2}}^{n-1} \cdots b_{j}-2 \cdots b_{k}, \quad \sum_{j=2}^{k} \quad b_{j}=n$; where $M W_{c_{1} c_{2}}^{n} \cdots c_{j} \cdots c_{k}=0$, if $c_{2}<0$. For $k=3$ the author's basic set gives a single formulation for the four types given by Protter (Portugaliae Mathematica vol. 10 (1951)); the differential recursion formulas correspond to those of Protter. (Received October 13, 1954.)

\section{E. J. Pellicciaro: Concerning critical sets of type 0.}

In a paper presented to the Society [Bull. Amer. Math. Soc. Abstract 59-1-61] 
the author proposed a classification of critical sets. The present paper concerns itself with critical sets of type 0 and proves the following theorem: Let $M=M(H, p)$ be a critical set of $f(x)$ of type 0 . If $q$ is a boundary point of $M$ belonging to $M$ and $U$ is a neighborhood of $q$, then $U$ contains a collection $T$ of critical points of $f(x)$ such that $T$ intersects an infinite number of critical sets of $f(x)$ and if $t$ belongs to $T$, then (i) $f(t)=f(p)$, (ii) for some neighborhood $u$ of $t, f(x) \leqq f(p)$ or $f(x) \geqq f(p)$ throughout $u$, and (iii) $t$ is a boundary point of some related complementary domain of $M(H, t)$. (Received October 13, 1954.)

\section{W. C. Royster: A coefficient problem for some classes of uni-} valent Laurent series.

Let $f(z)=\sum_{n=-\infty}^{g} a_{n} z^{n}, 0<\rho<|z|<1, a_{0}=0$, be regular and univalent for $\rho<|z|$ $<1$. Nehari and Schwarz (Proc. Amer. Math. Soc. vol. 5 (1954)) showed that if $f(z)$ belongs to the class of typically real Laurent series, the $\left|a_{n}\right|$ satisfy a sharp inequality and that if $f(z)$ belongs to the class of starlike functions represented by univalent Laurent series, the $\left|a_{n}\right|$ satisfy an inequality which was not sharp. In this paper the author improves the bound on $\left|a_{n}\right|$ for the starlikeness case; however, the bound again is not sharp. Sharp bounds are obtained for the class of functions which are starlike and satisfy the additional restriction that there is a real number $\mu$ such that $\operatorname{Im}\left\{f\left(\rho e^{i(\theta+\mu)}\right)\right\}$ and $\operatorname{Im}\left\{f\left(e^{i(\theta+\mu)}\right)\right\}$ change sign on the same ray. (Received October 13, 1954.)

\section{F. W. Stallard: Differential systems with interface conditions.} Preliminary report.

This paper considers systems of $n$ first order ordinary linear differential equations with two point boundary conditions and interface conditions at other points of the interval of the independent variable. Solutions, which ordinarily have discontinuities of the first kind at the interfaces, are studied. It is shown that there exist a discontinuous matrix, $D$, satisfying the interface conditions and $D^{\prime}(x)=0$, and an absolutely continuous matrix $W(x)$, satisfying an associated system, such that $D(x) W(x)$ is a solution of the given system and satisfies the boundary and interface conditions. The notion of a Green's matrix for such problems is introduced and its properties explored. (Received October 13, 1954.)

\section{George Swift: Irregular Borel measures on topological spaces.}

Let $X$ be a topological space, and let $\mathcal{B}, \mathcal{C}, \mathcal{V}$, and $\mathcal{W}$ be the classes of all Borel, compact, open, and open Borel sets of $X$, respectively. Recall that a Borel measure $\mu$ is outer (inner) irregular at a set $B \in \mathcal{B}$ when $\mu(B)<\inf \{\mu(\mathscr{W}): B \subset \mathscr{W} \in \mathscr{W}\}$ ( $>\sup \{\mu(C): B \supset C \in \mathcal{C}\}$ ). If $X$ is $\sigma$-compact and $\mu$ is totally finite, then $\mu$ is outer irregular at $B \in \mathbb{B}$ if and only if $\mu$ is inner irregular at $B^{\prime}$. Counterexamples exist for non- $\sigma$-compact $X$. Theorems concerning unions and differences of Borel sets at which $\mu$ is irregular are exhibited; in particular, if $\left\{A_{i}\right\}_{i=1}^{\infty} \subset \mathbb{B}$ are pairwise separable by sets of $\mathscr{W}, A=\bigcup_{i=1}^{\infty} A_{i}$, and $\mu(A)<\infty$, then $\mu$ is outer (inner) irregular at $A$ if and only if there exists an $i_{0}\left(i_{0}=1,2, \cdots\right)$ such that $\mu$ is outer (inner) irregular at $A_{i_{0}}$. This cannot be relaxed to pairwise disjoint $\left\{A_{i}\right\}_{i=1}^{\infty}$. Sums and differences of irregular measures are found to be irregular in certain cases; for example, if $\left\{\mu_{i}\right\}_{i=1}^{\infty}$ is a sequence of Borel measures on $X, \mu=\sum_{i=1}^{\infty} \mu_{i}$, and $\mu(X)<\infty$, then $\mu$ is a Borel measure on $X$ which is outer (inner) irregular at a set $B \in \mathcal{B}$ if and only if there exists an $i_{0}\left(i_{0}\right.$ $=1,2, \cdots)$ such that $\mu_{i}$ is outer (inner) irregular at $B$. Let $X$ be Hausdorff, let $Z$ be the set of all $x \in X$ such that $\mu(\{x\})>0$, and let $\mu(Z)<\infty$, then the set function 
$\psi$ defined by the relation $\psi(A)=\mu(A)-\mu\left(A \cap Z^{\prime}\right)$ for every $A \in \mathcal{B}$ is a Borel measure on $X$ such that (1) $\psi$ is outer (inner) irregular at $B \in \mathbb{B}$ if and only if $\mu$ is outer (inner) irregular at $B$, and (2) $\psi(\{x\})=0$ for every $x \in X$. (Received October 13,1954.)

\section{W. M. Zaring: Multiply monotone complex sequences.}

Denote by $\left\{c_{n}\right\}$ a null sequence of complex numbers. Then trivially $\Delta^{\alpha} c_{n}$ $\equiv \sum_{k=0}^{\infty} C_{k-\alpha-1, k} c_{n+k}$ converges for $\alpha \geqq 0$. If $\left|\arg \Delta^{\alpha} c_{n}\right| \leqq \phi<\pi / 2$, then we say that $\left\{c_{n}\right\}$ is $(\alpha, \phi)$-monotone. Using this definition of complex monotonicity some results of $\mathrm{K}$. Knopp, Mehrfach monotone Zahlenfolgen, Math. Zeit. vol. 22 (1925) pp. 75-85, and L. Fejer, Trigonometrische Reihen und Potenzreihen mit mehrfach monotone Koeffizientenfolge, Trans. Amer. Math. Soc. vol. 39 (1936) pp. 18-59, are extended to complex sequences. (Received October 13, 1954.)

\section{Applied Mathematics}

\section{R. G. Blake: Finite differences in laminated orthotropic plane strain.}

In order to obtain an approximate solution to the problem of plane strain in laminated orthotropic material, the differential equation is replaced by a set of finite equations. Stencils are then obtained for use in solving these equations by the relaxation method. (Received October 13, 1954.)

108t. Henry Gerhardt: Stresses in a catenoid membrane shell and the hyperbolic metric. Preliminary report.

A. Foppl in his Festigkeitslehre derives the differential equation of the stress function for a circular membrane. This paper attempts to extend his equation to the more general case of the catenoid membrane shell. A plane diagram of the stresses is given, using the hyperbolic rather than the euclidean metric. (Received October 11, 1954.)

\section{Nathaniel Macon: $A$ continued fraction for $e^{x}$.}

Using the continued fraction of Gauss, one can expand $e^{x}$ as a continued fraction $b_{0}+a_{1} /\left[b_{1}+a_{2} /\left(b_{2}+\cdots\right)\right]$, where $b_{0}=1, a_{1}=x, a_{2 n}=-x /[2(2 n-1)], a_{2 n+1}=x /[2(2 n$. $+1)]$, and $b_{n}=1(n=1,2,3, \cdots)$. It is shown that this expansion can be contracted to yield one in which $b_{0}=1, b_{1}=1-x / 2, a_{1}=x, b_{n}=2 n-1$, and $a_{n}=x^{2} / 4(n>1)$. This latter expansion converges quite rapidly and is well suited for digital computation. For example, if $-1 \leqq x \leqq 1$, one has $\left|e^{x}-A_{6} / B_{\theta}\right|<10^{-10}$. (Received October 14, 1954.)

\section{C. L. Seebeck, Jr. (p) and H. Hoelzer: Curve fitting using differential equations.}

The problem treated is: Given a set of points $\left(x_{j}, y_{j}\right)$, to find a differential equation plus initial conditions whose integral curve will approximate the given data. Linear homogeneous differential equations are used in a manner analogous to polynomials in the usual least square theory. The original set of $y_{j}$ are damped by multiplication by $e^{-\rho x}$. It is assumed that a curve $y=f(x)$ passes through this set of points and that its Laplace transform converges with its real part equal 0 . If $i w$ is its imaginary part, an identity in $w$ is established by applying the same Laplace transform to a given type of differential equation. Using this identity, the constants in the differential equation are determined in 3 different ways: 1 . Using computed points on the Laplace transform of a set of chords joining the given damped points; 2 . Using 
approximate initial conditions and moments of area of $f(x) ; 3$. Using only the moments of area. When linear homogenous differential equations are used, the techniques employed involve only function evaluation and solution of linear equations. Illustrations are given. (Received October 13, 1954.)

\section{GEOMETRY}

111. J. C. Morelock: $A$ note on the complete system of curves $|A|$ cut out on $F_{4}$.

In this paper it is shown by simple algebraic processes that the complete system of curves $|A|$ cut out on $F_{4}$ by all surfaces of order $P$ (where $P$ is a fixed prime) will in each case have certain characteristics, in fact: Theorem: $A$ necessary and sufficient condition that the complete system of curves $|A|$ (cut out of $F_{u}$ by all surfaces of order $P$ ) shall have its genus equal to its dimension is that $u=4$. (Received October 13,1954.)

112. J. C. Morelock and N. C. Perry ( $\mathrm{p})$ : A classification of algebraic surfaces invariant under cyclic collineations.

In algebraic geometry it is of interest to examine homogeneous polynomial surfaces of prime degree $p$ which transform into themselves under the collineation $T$ defined by $\left(x^{\prime}, x_{2}^{\prime}, x_{3}^{\prime}, x_{4}^{\prime}\right)=\left(x_{1}, E x_{2}, E^{2} x_{3}, E^{3} x_{4}\right)$ where $E^{p}=1$. A surface $F\left(x_{1}, x_{2}, x_{3}, x_{4}\right)$ $=0$ is invariant if each of its terms $x_{1}^{a} x_{2}^{b} x_{3}^{b} x_{4}^{d}$ is transformed by $T$ into the expression $E^{s} x_{1}^{a} x_{2}^{b} x_{3}^{b} x_{4}^{d}$ (where $s$ is of the same residue class (modulus $p$ ) for all terms). This property of a term is called s-invariance. The present paper proves the following results: Theorem: Necessary and sufficient conditions that $x_{1}^{a} x_{2}^{b} x_{3}^{c} x_{4}^{d}$ be $s$-invariant are that $a, b, c$, and $d$ are respectively members of the congruence classes $\bar{n}, \overline{r-2 n-s}$, $\overline{n-r+s} \bar{r}$ (determined by the parameters $n$ and $r$ ); and that $n$ and $r$ be restricted by certain lengthy inequalities which restrict the range of $n$ and $r$ as functions of $s$ and $p$. (Received October 13,1954.)

\section{T. L. Saaty: The number of vertices of a polyhedron.}

Relationship between convex polyhedra and a set of linear inequalities with a linear function to optimize is indicated. The number of steps involved in using iterative procedures for solving this type of problem, such as the simplex process, depend on the number of vertices of the convex set formed by the inequalities. It is desired to find upper bounds to the number of vertices as a function of the number of inequalities. Such an upper bound is determined, using Euler's formula and other auxiliary relations. It is found to be a polynomial of degree $(n-2)$ in the number of inequalities where $n$ is the number of the dimension dealt with. A theorem is given showing that an upper bound as a linear function of the number of inequalities $F_{n-1}$ dominated by the fundamental upper bound $F_{n-1} ! /\left(F_{n-1}-n\right) ! n$ ! does not exist for $n \geqq 4$. Generalizing this theorem using polynomials in $F_{n-1}$ would presumably show that the results obtained here cannot be further refined. (Received October 11, 1954.)

\section{H. C. Wang: A note on invariant affine connections.}

Let $G$ be a transitive group of differentiable homeomorphisms of a differentiable manifold $M$, and $H$ the isotropic subgroup at a point $p$ of $M$. Nomizu [Amer. J. Math. vol. 76 (1954) pp. 33-65] recently discussed and proved the existence of invariant affine connections II under $G$ when $G / H$ is a reductive homogeneous space. In this note, the author gives a both necessary and sufficient condition for the exist- 
ence of such a $\Pi$. In fact, let $\&$ denote the tangent space of $M$ at $p$, and $\tau$ the natural linear representation of $H$ over $\&$. Then there exists an affine connection over $M$ invariant under $G$ if and only if $\tau$ is faithful. Moreover, if there exists one, there must exist a symmetric one. The proof consists in merely geometrical arguments about the bundle of affine frames over $M$. (Received October 13, 1954.)

\section{TOPOLOGX}

\section{R. W. Bagley: A maximal set in the lattice of topologies.}

Let $\Lambda$ be the lattice of $T_{1}$ topologies on an infinite set. The ordering in $\Lambda$ is defined as follows: $\alpha \leqq \beta$ whenever each set open under $\beta$ is also open under $\alpha$. Let $\Lambda_{0}$ be the subset of $\Lambda$ (with the same ordering) consisting of all elements which are lattice products of hyperplanes in addition to the last element of $\Lambda_{.} \Lambda_{0}$ is a full set algebra and is maximal with respect to containing unique complements and finite lattice products. (Received October 8, 1954.)

\section{T. R. Brahana: The local Betti numbers in topological product} spaces.

The local Betti numbers $p^{r}(x, y), r=0,1, \cdots$ (see Wilder, Topology of manifolds), of points $(x, y)$ in the topological product $X \times Y$ of two locally compact topological spaces $X$ and $Y$ can be determined by knowledge of the local Betti numbers $p^{8}(x)$ and $p^{t}(y)$ of the projections of the points on the factor spaces, by the formula $p^{r}(x, y)$ $=\sum_{s+t-r} p^{s}(x) p^{t}(y)$, which is established in this paper. The proof depends on the Cech homological equivalence of the following form: $H^{r}(X \times Y, X \times B \cup A \times Y)$ $\approx \sum_{s+t=r} H_{s}(X, A) \otimes H_{t}(Y, B)$, where $(X, A)$ and $(Y, B)$ are compact pairs. Application of the formula above is made to prove that the topological product of generalized manifolds (subject to mild restrictions) is again a generalized manifold; the more general statement holds: the bundle space of a fibre bundle whose base and fibre are in the class of generalized manifolds referred to is again a generalized manifold. Application of the theorem is made to the problem of dimension of product spaces. (Received September 29, 1954.)

\section{C. E. Capel: Space of subsets and cartesian products.}

For a space $X$, let $S(X)$ denote the space of all non-void, closed subsets of $X$ with the Frink topology. (If $U$ and $V$ are open subsets of $X$ then the collection of all closed sets contained in $U$ and meeting $V$ is a subbasis element of this topology.) Let $\left\{X_{a}\right\}$ be a collection of spaces with $P\left\{X_{a}\right\}$ and $P\left\{S\left(X_{a}\right)\right\}$ denoting the cartesian products; then $P\left\{S\left(X_{a}\right)\right\}$ can be considered as a subset of $S\left(P\left\{X_{a}\right\}\right)$. It is shown that if the projections $p_{a}: P\left\{X_{a}\right\} \rightarrow X_{a}$ are closed functions, then for any space $Y$ and any continuous function $f: P\left\{S\left(X_{a}\right)\right\} \rightarrow Y$ there is a continuous extension $f^{*}$ : $S\left(P\left\{X_{a}\right\}\right) \rightarrow Y$. Hence $P\left\{S\left(X_{a}\right)\right\}$ is a retract of $S\left(P\left\{X_{a}\right\}\right)$. (Received October 15, 1954.)

\section{W. M. Faucett and R. J. Koch (p): Complements of maximal} ideals in compact semigroups.

Let $S$ denote a compact Hausdorff topological semigroup. It is known that $S$ contains maximal proper ideals, necessarily open. Let $J$ be a maximal proper ideal of $S$; it is shown that $S / J$ (Rees quotient) is either the trivial semigroup of two elements or else is completely simple [Rees, Proc. Cambridge Philos. Soc. vol. 36 (1940) pp. 387-400]. It follows that the complement of $J$ is the disjoint union of compact iso- 
morphic groups and of sets whose square lies in $J$. The equivalence between regular and simple elements [J. A. Green, Ann. of Math. vol. 54 (1951) pp. 163-172] in a compact semigroup is demonstrated. The maximal subgroup containing an idempotent $e$ is characterized as the set of bi-ideal [Good and Hughes, Bull. Amer. Math. Soc. Abstract 58-6-575] generators of eSe. Maximal elements are defined and studied. A forthcoming result of R. J. Koch and A. D. Wallace is generalized as follows: if $S^{2}=S$, then $S=S \widetilde{E} S$ where $\widetilde{E}$ is a set of idempotents chosen one from the complement of each maximal proper ideal. (Received October 7, 1954.)

\section{W. L. Gordon: Separating sets in Euclidean spaces.}

The following theorem, upon application to compact subsets $X$ of Euclidean $n+2$ space, provides sufficient intrinsic conditions for $X$ to cut $R^{n+2}$, thus yielding a generalization and variation of a classical theorem of Janiszewski. Let $X$ be paracompact with $A$ and $B$ closed subsets satisfying $X=A \cup B$ and the homomorphism $i^{*}: H^{n}(A) \rightarrow H^{n}(A \cap B)$ (the $n$th Alexander-Kolmogoroff cohomology groups, coefficients arbitrary) not onto. If either (a) $H^{n}(B)=0$ or (b) there is a continuous mapping of $A$ onto $B$ whose restriction to $A \cap B$ is deformable in $B$ to the identity map of $A \cap B$, then $H^{n+1}(X)$ is not zero. (Received October 13, 1954.)

\section{J. S. Griffin, Jr.: Intrinsic systems of covering paths.}

Let $B=\{B, X, \pi, F, \mathcal{V}, \phi, G\}$ be a fibre bundle and let $\mathcal{P}$ be a family of paths in $X$. Families $\mathcal{F}$ subject to the following condition are studied: for each $x \in X$ and $p \in \mathcal{P}$ such that $\pi(x)=p(t)$, there is a unique $q \in \mathcal{F}$ such that $q(t)=x$ and $\pi q=p$. Since $\mathcal{F}$ is a function space, there are natural topological restrictions; in particular, in the presence of suitable hypotheses $\mathcal{F}$ may be made to yield covering homotopies. In this case, $\mathcal{F}$ gives a family of paths in the space $B^{F}$ and thus it is natural to consider the associated principal bundle, or more generally any associated bundle. Finally such an $\mathcal{Z}$ may be shown to be equivalent to a family of paths in $G$. On the one hand this notion includes the families of (unique) covering paths for $F$ totally disconnected, and the families of (unique in a sense) covering paths for $G$ totally disconnected. On the other, if $B$ is the tangent bundle for a differentiable manifold and $P$ is the family of all differentiable paths, then an affine connection for $X$ is equivalent to a family $\mathcal{F}$. (Received November 29,1954 .)

121. O. G. Harrold, H. C. Griffith, and E. E. Posey (p): The imbedding in three-space of cells which are locally polyhedral except at boundary points.

In this paper the authors give a new proof of their theorem on a characterization of tamely imbedded curves in three-space (Bull. Amer. Math. Soc. Abstract 60-4-542. To appear in Trans. Amer. Math. Soc.). The proof of the present paper is direct in the sense that a simple closed curve in three-space that has property $\mathrm{P}$ and which bounds a topological 2-cell that is locally polyhedral modulo the boundary is approximated "uniformly" by a sequence of unknotted polygonal simple closed curves. The essential construction is that of a sequence of piecewise linear homeomorphisms on threespace whose limit is a homeomorphism. (Received October 13,1954.)

\section{S. T. Hu: On differential forms in differentiable spaces.}

As an obvious generalization of differentiable manifolds of class $C^{k}$, the author defined in 1949 the notion of the differentiable spaces of class $C^{k}$ (See Ann. of Math. vol. 50, p. 266). A subset $M$ of a euclidean space $E^{n}$ is said to be a differentiable space 
of class $C^{k}$ if there exist an open set $U$ of $E^{n}$ containing $M$ and a differentiable mapping $r$ : $U \rightarrow M$ of class $C^{k}$ such that $r(x)=x$ for each $x$ in $M . r$ is called a retraction of class $C^{k}$. In the present paper, the differential forms in a differentiable space are defined in a natural way and the theorems of de Rham are established for this generalization. However, the main interest of the work is not the generalization but the simplification of the classical case by getting rid of the clumsy systems of local coordinates. (Received October 13, 1954.)

\section{I. S. Krule: On ordered topological spaces. Preliminary report.}

Let $X$ be a Hausdorff space, and let $L$ be a nonvoid closed transitive subset of $X \times X$. If $A \subset X$, let $L(A)=\{x \in X \mid(x, a) \in L$ for some $a \in A\} . L$ is monotone if $L(x)$ is connected for each $x \in X . L$ is continuous if $L\left(A^{*}\right) \subset L(A)^{*}$ for each $A \subset X$. If $X$ is a continuum and $L$ is continuous, then for each $x \in X, L(x) \neq \varnothing$, and $X$ contains reflexive elements. Using definitions and techniques analogous to those used by Wallace and Koch in the study of topological semigroups, generalizations and order analogues of results in topological semigroups are obtained quite easily. For example, if $X$ is a metric indecomposable continuum and $L$ is reflexive, monotone, and continuous, then every element in $X$ is $L$-minimal (cf. Wallace, Math. J. of Okayama Univ. vol. 3 (1953) p. 1). Examples are obtained which show that the analogy considered between topological semigroups and ordered spaces is incomplete. (Received October 13, 1954.)

124t. R. L. Plunkett: A note on convex metrics with unique segments. Preliminary report.

It is pointed out by R. H. Bing [A convex metric with unique segments, Proc. Amer. Math. Soc. vol. 4 (1953) pp. 167-174] that every continuous curve which has a convex metric with unique segments is contractible and locally contractible and examples are given of spaces which fail to have a convex metric with unique segments because of failure to be contractible, in one case, and locally contractible, in another. In this note, it is proved that each continuous curve which has a convex metric with unique segments is an absolute retract for separable metric spaces. Thus, the example by Borsuk [Sur un espace compact locally contractile qui est n'est pas un rêtracte absolu de voisinage, Fund. Math. vol. 35 (1948) pp. 175-180] of a compact space which is contractible and locally contractible but which is not an absolute retract affords an example of a space which does not have a convex metric because it fails to be an absolute retract, although it is both contractible and locally contractible. (Received October 13, 1954.)

\section{W. L. Strother: A new characterization of Peanian spaces.}

Hahn and Mazurkewicz showed that a compact metric space $X$ is a Peanian space if and only if $X$ is a locally connected continuum. This paper shows that a metric space $X$ is a Peanian space (continuous image of the unit interval) if and only if $X$ is homeomorphic to a multi-retract of a Tychonoff cube. ( $A$ is said to be a multi-retract of $X$ if there exists a continuous multi-valued function $F$ on $X$ to $A$ such that $F(a)=a$ for all $a$ in $A$.) (Received October 15, 1954.)

126t. P. M. Swingle: Locally $n$-indecomposable and related concepts.

Let $R$ be a region, $x \in R ; W$ has a local essential sum at $x$ w.r.t. $R$ of the class $(C)$ of subconnexes of $W$ if there exists a region $R^{\prime}$ in $R, x \in R^{\prime}$, such that $W \cdot R^{\prime} C U(C)$ 
but no $C \cdot R^{\prime}$ is contained in the closure of the sum of the other $C \cdot R^{\prime \prime}$ s. A connexe $W$ is locally $n$-indecomposable at $x$ if, for every region $R, x \in R, W$ has a local essential sum at $x$ w.r.t. $R$ of a class of $n$, but not more than $n$, subconnexes of $W$. If $n$ is an integer $>1$, there does not exist a connexe $W$ which is locally $n$-indecomposable at each of its points; also in a completely separable space there does not exist a connexe $W$ which is locally finitely-indecomposable at each of its points, but then for $x \in$ connexe $W, W$ at $x$ either is locally (aleph-zero)-indecomposable, or locally finitelyindecomposable, or there exists an integer $n_{x}>0$ such that $W$ is locally $n_{x}$-indecomposable there. If (1) the space also is compact, (2) $W$ is locally $n$-indecomposable at $x \in W$, but (3) not locally (aleph-zero)-indecomposable at any point of the closure of $W$, then $W$ is locally $n$-irreducible at $x$; also, if (1) and (3), $W$ is locally $n$-indecomposable at $x$ if and only if, for every region $R, x \in R, W$ has a local essential sum at $x$ w.r.t. $R$ of a class (I) of $n$ indecomposable subconnexes of $W$. (Received March 11, 1954.)

\section{A. D. Wallace: Struct ideals on compact spaces.}

A struct on a space $X$ is a nonvoid closed and transitive subset of $X \times X$. If $A \subset X$ let $L(A)$ be all $x$ such that $(x, a) \in L$ for some $a \in A$, where $L$ is a struct on $X$. The set $A$ is an $L$-ideal if $A \neq \square$ and if $L(A) \subset A$. We let $L_{0}(A)$ be the union of $L$ ideals contained in $A$. Lemma. If $X$ is compact and if $U$ is open, then $L_{0}(U)$ is open. Theorem. Let $X$ be compact, let $A$ be an $L$-ideal, and let $B$ be a closed set with $B$ not contained in $A$. There exists an $L$-ideal maximal among all $L$-ideals that contain $A$ and do not contain $B$ and each such is open. If $a \in X$ let $L_{a} \neq\{x \mid a \cup L(a)=x \cup L(x)\}$. One defines $L$-maximal and $L$-minimal elements. The complements of sets $L_{a}$ with $a L$-maximal and $L_{a} \neq X$ are maximal $L$-ideals and conversely. If $X$ is compact and if $K$ is the set of $L$-minimal elements, then $K$ is the union of all minimal $L$-ideals. If $X$ is compact and if $L$ satisfies also $L\left(A^{*}\right) \subset L(A)^{*}$ for all $A$, then $K$ is closed. (Received July 26,1954 .)

\section{R. F. Williams: $A$ note on unstable homeomorphisms.}

An example of an unstable homeomorphism of a compact metric continuum (the "two-solenoid") onto itself is given. This answers a question raised by W. R. Utz (Proc. Amer. Math. Soc. vol. 1 (1950) pp. 769-774) who defined the term unstable homeomorphism in the same paper: a homeomorphism $f$ of a metric space $X$ onto $X$ is said to be unstable provided that there exists a fixed positive number $\delta$, such that if $x$ and $y$ are distinct points of $X$, then there exists an integer $n$, such that $\rho\left[f^{n}(x), f^{n}(y)\right]$ $>\delta$. (This research was sponsored by The National Research Foundation under contract NSF-G358.) (Received June 28, 1954.)

129. C. T. Yang: Mappings from spheres to euclidean spaces. II.

As a continuation of recent works of the author (Bull. Amer. Math. Soc. Abstracts 59-6-720 and 60-4-547) it is proved that a mapping of a $(3 \mathrm{~m})$-sphere into the euclidean $m$-space maps the end points of some 3 mutually orthogonal diameters into a single point. (Received October 14, 1954.)

J. H. ROBERTS, Associate Secretary 